\title{
GENERALIZED PRIME IDEALS IN NON-ASSOCIATIVE NEAR-RINGS I
}

\author{
YONG UK CHO
}

\begin{abstract}
In this paper, the concept of $*$-prime ideals in non-associative near-rings is introduced and then will be studied. For this purpose, first we introduce the notions of $*$-operation, $*$-prime ideal and $*$-system in a near-ring. Next, we will define the $*$-sequence, $*$-strongly nilpotent and $*$-prime radical of near-rings, and then obtain some characterizations of $*$-prime ideal and *-prime radical $r_{s}(I)$ of an ideal $I$ of near-ring $N$.
\end{abstract}

\section{Introduction}

A near-ring $N$ is an algebraic system $(N,+, \cdot)$ with two binary operations, say + and $\cdot$ such that $(N,+)$ is a group (not necessarily abelian) with neutral element $0,(N, \cdot)$ is a semigroup and $a(b+c)=a b+a c$ for all $a, b, c$ in $N$.

In this near-ring, if $(N, \cdot)$ is not a semigroup, then $N$ is a non-associative near-ring. If $N$ has a unity 1 , then $N$ is called unitary. An element $d$ in $N$ is called distributive if $(a+b) d=a d+b d$ for all $a$ and $b$ in $N$. A near-ring $N$ is called distributive if every element in $N$ is distributive.

An ideal of $N$ is a subset $I$ of $N$ such that (i) $(I,+)$ is a normal subgroup of $(N,+)$, (ii) $a(I+b)-a b \subset I$ for all $a, b \in N$, (iii) $(I+a) b-a b \subset I$ for all $a, b \in N$. If $I$ satisfies (i) and (ii) then it is called a left ideal of $N$. If $I$ satisfies (i) and (iii) then it is called a right ideal of $N$.

On the other hand, an $N$-subgroup of $N$ is any subset $H$ of $N$ such that (i) $(H,+)$ is a subgroup of $(N,+)$, (ii) $N H \subset H$ and (iii) $H N \subset H$. If $H$ satisfies (i) and (ii) then it is called a left $N$-subgroup of $N$. If $H$ satisfies (i) and (iii) then it is called a right $N$-subgroup of $N$. In case, $(H,+)$ is normal in above, we say that normal $N$-subgroup, normal left $N$-subgroup and normal right $\mathrm{N}$-subgroup instead of $\mathrm{N}$-subgroup, left $\mathrm{N}$-subgroup and right $\mathrm{N}$-subgroup, respectively.

Note that normal $N$-subgroups of $N$ are not equivalent to ideals of $N$.

We consider the following notations: Given a near-ring $N$,

$$
N_{0}=\{a \in N \mid 0 a=0\}
$$

Received June 7, 2011; Revised September 25, 2011; Accepted April 25, 2012.

2000 Mathematics Subject Classification. 16 Y30.

Key words and phrases. Non-associative near-rings, *-prime ideal, *-system, *-strongly nilpotent and $*$-prime radical. 
which is called the zero symmetric part of $N$,

$$
N_{c}=\{a \in N \mid 0 a=a\}=\{a \in N \mid r a=a, \text { for all } r \in N\}
$$

which is called the constant part of $N$.

We note that $N_{0}$ and $N_{c}$ are subnear-rings of $N$. A near-ring $N$ with the extra axiom $0 a=0$ for all $a \in N$, that is, $N=N_{0}$ is said to be zero symmetric, also, in case $N=N_{c}, N$ is called a constant near-ring. From the Pierce decomposition theorem, we get the important fact:

$$
N=N_{0} \oplus N_{c}
$$

as additive groups. So every element $a \in N$ has a unique representation of the form $a=b+c$, where $b \in N_{0}$ and $c \in N_{c}$.

Throughout this paper, by a near-ring, we mean a zero-symmetric nonassociative near-ring. For basic definitions and results on near-rings, one may refer Pilz [5].

Let $(G,+)$ be a group (not necessarily abelian). In the set

$$
M(G)=\{f \mid f: G \longrightarrow G\}
$$

of all the self maps of $G$, if we define the sum $f+g$ of any two mappings $f, g$ in $M(G)$ by the rule $x(f+g)=x f+x g$ for all $x \in G$ and the product $f \cdot g$ by the rule $x(f \cdot g)=(x f) g$ for all $x \in G$, then $(M(G),+, \cdot)$ becomes a near-ring. It is called the self map near-ring of the group $G$. Also, if we define the set

$$
M_{0}(G)=\{f \in M(G) \mid 0 f=0\},
$$

then $\left(M_{0}(G),+, \cdot\right)$ is a zero symmetric near-ring.

\section{Results on $*$-prime ideals and $*$-prime radicals}

Groenewald and Potgieter [1] generalized the notion of prime ideals in associative near-rings and introduced the concept of $f$-prime ideals in associative near-rings. The notion of $f$-prime ideals in associative near rings actually extends the notion of $f$-prime ideals in associative rings due to Murata et al. [2]. Myung [3] introduced the notion of $*$-prime ideals in non-associative rings. Corresponding to $*$-prime ideals in non-associative rings, we can introduce in this paper the $*$-prime ideals in non-associative near-rings. For this purpose, first we define the notions of $*$-system and $*$-prime ideal in a near-ring and prove that complement of a $*$-system is a $*$-prime ideal.

In this section, we define $*$-operation for the purpose of $*$-prime ideals, and obtain some characterizations of $*$-prime ideal and $*$-prime radical.

The concept of $*$-operation for rings was introduced by Myung [3], [4]. We can extend this concept to near-rings as following:

Definition 1. Let $F(N)$ be the set of all ideals in $N$. A *-operation is a mapping from $F(N) \times F(N)$ into the family of additive subgroups of $N$ satisfying the following conditions.

(i) for $A, B, C, D$ in $F(N)$, if $A \subseteq B$ and $C \subseteq D$, then $A * C \subseteq B * D$. 
(ii) $A * B \subseteq A \cap B$ for all $A, B$ in $F(N)$.

(iii) $(A+C) *(B+C) \subseteq(A * B)+C$ for all $A, B, C$ in $F(N)$.

Hereafter, by a near-ring we mean a near-ring $N$ in which a $*$-operation is defined.

Now, we may obtain the following examples of $*$-operations in $N$.

Example 1. Let $N$ be a near-ring. Define $*$ on $F(N) \times F(N)$ by $A * B$ is a normal subgroup generated by $\{a b \mid a \in A, b \in B\}$. Then this $*$-operation satisfy the conditions stated in the above Definition 1 . For, the conditions $(i)$ and (ii) are trivially true. If $A, B, C \in F(N)$, then $(A+C)(B+C) \subseteq A B+C$. Thus the set of all generators of $(A+C) *(B+C)$ are of the form $a b+c$ for $a \in A, b \in B$ and $c \in C$. Clearly $A * B+C$ is a normal subgroup of $(N,+)$ and it contains all the elements of $A B+C$. Thus $(A+C) *(B+C) \subseteq A * B+C$. Hence for any near-ring $N$, always $*$-operation exists.

Definition 2. A proper ideal $I$ in a near-ring is said to be $*$-prime if $A * B \subseteq I$ implies either $A \subseteq I$ or $B \subseteq I$ for $A, B$ in $F(N)$.

Definition 3. A non-empty subset $M$ of $N$ is said to be $*$-system if $A \cap M \neq \emptyset$ and $B \cap M \neq \emptyset$ implies $A * B \cap M \neq \emptyset$ for $A, B \in F(N)$.

In the following, we give some examples of $*$-prime ideals in $N$.

Example 2. Consider the near-ring $(N,+,$.$) defined on Dihedral group$ $\left(D_{8},+\right)$ according to the scheme $(0,9,0,9,1,3,1,3)($ p. $415[5])$. This near-ring is non-associative, since $(a+b)((2 a+b)(3 a+b))=a+b$ and $((a+b)(2 a+b))(3 a+$ $b)=3 a+b$. One can check that the proper ideals of the above near-ring are $I_{1}=\{0,2 a\}$ and $I_{2}=\{0, a, 2 a, 3 a\}$. This follows from the fact that the above near-ring is distributive and $I_{1}$ and $I_{2}$ are the only normal subgroups which are closed under left and right multiplications by elements of $N$. Define $*$ on $F(N) \times F(N)$ as in Example 1. For this $*$-operation, it is easy to observe that $I_{2}$ is $*$-prime and $I_{1}$ is not a $*$-prime ideal in $N$.

Now, we can obtain some equivalent conditions of $*$-prime ideals in $N$.

Proposition 2.1. Let I be a proper ideal in a near-ring $N$. Then the following are equivalent:

(i) If $A * B \subseteq I$ for $A, B$ in $F(N)$, then either $A \subseteq I$ or $B \subseteq I$.

(ii) If $A \cap C(I) \neq \emptyset$ and $B \cap C(I) \neq \emptyset$, then $(A * B) \cap C(I) \neq \emptyset$ for $A, B \in F(N)$. Here $C(I)$ denotes complement of $I$.

(iii) If $a$ and $b$ are in $C(I)$, then $(\langle a\rangle *\langle b\rangle) \cap C(I) \neq \emptyset$, where $\langle x\rangle$ denotes the ideal generated by $x \in N$.

Proof. (i) $\Rightarrow$ (ii). Assume the condition (i). If $A \cap C(I) \neq \emptyset$ and $B \cap C(I) \neq \emptyset$, then there exist $a$ in $A$ and $b$ in $B$ such that $a \in C(I)$ and $b \in C(I)$, that is, $a \notin I$ and $b \notin I$. These fact implies that $A \nsubseteq \subseteq I$ and $B \nsubseteq I$. From the condition (i), we see that $A * B \nsubseteq I$, that is, there exists $c \in(A * B)$ such that $c \notin I$, equivalently, there exists $c \in(A * B)$ such that $c \in C(I)$. Hence, $(A * B) \cap C(I) \neq \emptyset$ for $A, B \in F(N)$. 
$(i i) \Rightarrow(i i i)$ and $(i i i) \Rightarrow(i)$ can be, analogously, proved as $(i) \Rightarrow(i i)$.

Remark 1. By the above Proposition 2.1, an ideal $I$ is a $*-$ prime ideal if and only if $C(I)$ is a $*$-system. Thus in Example 2, the set $M=\{b, a+b, 2 a+$ $b, 3 a+b\}$ is a $*$-system.

Definition 4. A sequence $a_{0}, a_{1}, \ldots, a_{n}, \ldots$ of elements in $N$ is said to be a *-sequence if $a_{n} \in<a_{n-1}>*<a_{n-1}>$ for all $n \geq 1$.

Lemma 2.2. Every $*$-sequence is a $*$-system in $N$.

Proof. Let $S=\left\{a_{0}, a_{1}, \ldots, a_{n}, \ldots\right\}$ be a $*$-sequence in N. If $A \cap S \neq \emptyset$ and $B \cap S \neq \emptyset$, then there exist elements $a_{k}$ and $a_{\ell}$ in $S$ such that $a_{k} \in A$ and $a_{\ell} \in B$. If $k \geq \ell$, then $a_{k+1} \in<a_{k}>*<a_{k}>\subseteq<a_{k}>*<a_{\ell}>\subseteq A * B$ and so $(A * B) \cap S \neq \emptyset$. Thus $S$ is a $*$-system in $N$.

Definition 5. An element $a \in N$ is said to be $*$-strongly nilpotent if every *-sequence $a_{0}, a_{1}, \ldots, a_{n}, \ldots$ with $a_{0}=a$ vanishes. That is, there exists an integer $k \geq 1$ such that $a_{s}=0$ for all $s \geq k$.

Definition 6. If $I$ is a proper ideal of $N$, then the $*$-prime radical $r_{S}(I)$ of $I$ is the set of all elements $x \in N$ such that every $*$-system that contains $x$ contains an element of $I$.

Proposition 2.3. For an ideal $I$ of a near-ring $N, r_{S}(I)$ is the intersection of all $*$-prime ideals in $N$ containing $I$.

Proof. Let $x \in r_{S}(I)$. Suppose $x \notin \cap P_{i}$, where $P_{i}$ is a $*$-prime ideal containing $I$. By assumption there exists a $*$-prime ideal $P$ such that $x \notin P$ and $I \subseteq P$. Since $P$ is a $*$-prime ideal, $C(P)$ is a $*$-system containing $x$ and $C(P) \cap I=\emptyset$. This is a contradiction. Hence $r_{S}(I) \subseteq \cap P_{i}$.

Conversely, if $x \in \cap P_{i}$ and $x \notin r_{S}(I)$, then there exists a $*$-system $\mathrm{M}$ such that $x \in M$ and $M \cap I=\emptyset$. This implies that $C(M)=P$ is a $*$-prime ideal and $x \notin P$, a contradiction. Thus $\cap P_{i} \subseteq r_{S}(I)$

Proposition 2.4. Let $N$ be a near-ring. Then $r_{S}(N)=\{n \in N / n$ is $*$-strongly nilpotent \}.

Proof. Let $x \in r_{S}(N)$. If $x$ is not $*$-strongly nilpotent, then there exists a *-sequence $S=\left\{a_{0}, a_{1}, \ldots, a_{n}, \ldots\right\}$ with $a_{0}=x$ and $a_{n} \neq 0$ for all $n \geq 1$. By Lemma 2.2, $S$ is a $*$-system. Again by Proposition 2.1, $C(S)$ is a *-prime ideal and note that $x \notin C(S)$. Thus $x \notin r_{S}(N)$, a contradiction.

Conversely let $x$ be a $*$-strongly nilpotent. If $x \notin r_{S}(N)$, then there exists a $*$-prime ideal $P$ such that $x \notin P$. By Proposition 2.1, $C(P)$ is a $*$-system and $x \in C(P)$. Since $a_{0}=x \in<x>\cap C(P)$, by the definition of $*$-system we get $\left(<a_{0}>*<a_{0}>\right) \cap C(P) \neq \emptyset$. Let $a_{1} \in\left(<a_{0}>*<a_{0}>\right) \cap C(P)$. Since $<a_{1}>\cap C(P) \neq \emptyset$ we get an element $a_{2} \in\left(<a_{1}>*<a_{1}>\right) \cap C(P)$. Continuing in this way we get a $*$-sequence $S=\left\{a_{0}, a_{1}, \ldots\right\}$ with $a_{0}=x$. Note that $S \subseteq C(P)$. By the assumption, $x$ is $*$-strongly nilpotent, there exists 
an integer $k \geq 1$ such that $a_{s}=0$ for all $s \geq k$. Thus $a_{k}=0 \in P$ and so $P \cap C(P) \neq \emptyset$, a contradiction. Thus $x \in r_{S}(N)$.

\section{References}

[1] N. J. Groenewald and P. C. Potgieter A generalization of prime ideals in near-rings, Comm. in Algebra 12(15) (1984), 1835-1853.

[2] K. Murata, Y. Murata and K. Marubayashi A generalization of prime radical in rings, Osaka J. Math. 66 (1969), 291-301.

[3] H. C. Myung On prime ideals and primary decomposition in a non-associative rings, Osaka J. Math. 9 (1972), 41-47.

[4] H. C. Myung A generalization of prime radical in non-associative rings, Pacific Journal of Mathematics 42 (1) (1972), 187-193.

[5] G. Pilz Near-rings, , North Holland Publishing Company, Amsterdam, New York, Oxford 1983.

YONG UK CHO

Department of Mathematics, College of Education, Silla University, Pusan 617736, KOREA

E-mail address: yucho@silla.ac.kr 